Vol. $8 \mid 20$ | International Journal of

№2 21 Medical and Surgical Sciences

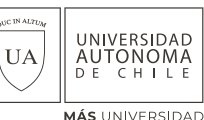

Article

UBICAGIÓN INTRAHOSPITALARIA Y

TRATAMIENTO ANTIMIGROBIANO INIGIAL

EN PAGIENTES GON NEUMONÍA ADQUIRIDA

EN LA GOMUNIDAD Y SU RELAGIÓN GON LA

FREGUENGIA Y GALIDAD DEL PROGESO DE

ESTRATIFIGAGIÓN

\title{
Intrahospitalary location and antimicrobial initial treatment in patients with acquired pneumonia in the community and their relationship with the frequency and quality of the stratification process
}

Luis Alberto Corona Martínez (D)
Servicio de Medicina Interna, Hospital Universitario "Dr Gustavo Aldereguía Lima”, Cienfuegos, Cuba.

Iris GonzÁlez Morales (D)

Servicio de Medicina Interna, Hospital Universitario "Dr Gustavo Aldereguía Lima”, Cienfuegos, Cuba.

\author{
María Caridad Fragoso Marchante (D) \\ Servicio de Medicina Interna, Hospital Universitario “Dr Gustavo Aldereguía Lima”, Cienfuegos, Cuba.
}

Autor de correspondencia: Luis Alberto Corona Martínez.

Calle 63 No. 607a, entre 6 y 8 NE. Cienfuegos. Provincia de Cienfuegos. Cuba.

Correo electrónico: luis.corona@gal.sld.cu

Recibido: $21 / 12 / 2020$

Aceptado: 02/02/2021

\section{RESUMEN}

La importancia de la evaluación inicial de la gravedad del paciente con neumonía es una acción diagnóstica de importancia bien establecida. El objetivo del trabajo fue evaluar la relación entre la frecuencia y calidad del proceso de estratificación de pacientes con neumonía, y el cumplimiento de las sugerencias de ubicación intrahospitalaria y de tratamiento antimicrobiano inicial de un instrumento de estratificación. Se realizó un estudio descriptivo sobre una población de 1,809 pacientes hospitalizados durante 10 años. Se analizó el comportamiento de los índices de ubicación intrahospitalaria y tratamiento antimicrobiano inicial acorde a la sugerencia de un instrumento de estratificación utilizado; en el análisis estadístico se utilizó el Odds ratio y el estadígrafo $\mathrm{X}^{2}$, con 
un nivel de significación de 95\%. En los resultados se destacan que la ubicación intrahospitalaria estuvo acorde a la sugerencia del instrumento en el $96 \%$, con el valor más bajo en los pacientes con neumonía grave y altas probabilidades de recuperación $(82 \%, \mathrm{p}<, 05)$. Se constató mayor frecuencia de ubicación intrahospitalaria acorde a la sugerencia del instrumento en los pacientes bien estratificados $(\mathrm{p}<, 05)$, fundamentalmente en los pacientes con neumonía grave y altas probabilidades de recuperación. La correspondencia del tratamiento antimicrobiano inicial con la propuesta del instrumento fue del $61 \%$; el estrato IIIA mostró el valor más elevado $(80 \%$, $\mathrm{p}<, 05)$. Como conclusiones del estudio se constató un elevado desempeño en el cumplimiento de la sugerencia de ubicación intrahospitalaria del instrumento de estratificación, no así en el cumplimiento de la sugerencia de tratamiento antimicrobiano inicial. Se demostró la existencia de una relación entre el proceso de estratificación y el cumplimiento de la ubicación intrahospitalaria sugerida por el instrumento empleado.

Palabras clave: neumonía. Infecciones comunitarias adquiridas. Ingresos hospitalarios.

\section{Introducción}

Las infecciones agudas del tracto respiratorio inferior, y la neumonía adquirida en la comunidad (NAG) en particular, constituyen una causa frecuente de consulta médica en los servicios de urgencias y ambulatorios, así como de ingresos hospitalarios, tanto a nivel mundial, como en Cuba y en el contexto de la provincia de Cienfuegos (Báez et al., 2013; Huerta et al., 2013; Spoorenberg et al., 2014; Ministerio de Salud Pública, 2019; Julián et al., 2018).

En Cuba, en particular, esta afección ha ocupado el quinto lugar entre las principales causas de muerte desde el año 1995, y a partir del año 2001 constituye la cuarta causa junto con la infección por influenza; tanto desde el punto de vista del número total de defunciones que aporta, como por la tasa de mortalidad bruta y ajustada, convirtiéndose de este modo también en la principal causa de muerte de origen infeccioso (Ministerio de Salud Pública, 2019).

Está reconocida la importancia de la evaluación inicial de la gravedad del paciente con NAC como paso clave para establecer la ubicación más apropiada para su cuidado y el tratamiento con antimicrobianos (Falguera \& Ramírez, 2015; Martínez et al., 2020; Aleaga et al., 2015; Calle et al., 2014).En este sentido, varias publicaciones sobre la estratificación inicial de pacientes con NAC recomiendan la utilización de escalas pronosticas de gravedad, cuyo propósito es clasificar a los pacientes en diferentes grupos de riesgo en función de la probabilidad de fallecer. En la mayoría de los países, incluyendo Cuba, las escalas más utilizadas han sido el CURB-65 y el Índice de severidad de la neumonía (PSI, por sus siglas en inglés) (Fine et al., 1997; Torres et al., 2013).

En nuestra institución se ha estado aplicando una herramienta para la estratificación del paciente con neumonía asistido en el Departamento de urgencias desde hace más de una década. Esta herramienta, denominada "Instrumento para la Estratificación del paciente con neumonía adquirida en la comunidad" (IENAC) (Corona, et al., 2015) y presentada en el anexo 1, ha sido desarrollada con el objetivo principal de conducir al médico asistencial en la toma de decisiones de conducta y de manejo terapéutico, específicamente en lo que se refiere al antimicrobiano inicial a indicar.

El prolongado tiempo transcurrido en la utilización del IENAC justifica la evaluación de indicadores del proceso efectuado. Por tanto, el objetivo del trabajo ha sido evaluar la relación entre la frecuencia de realización y la calidad del proceso de estratificación de pacientes con NAC y el cumplimiento de las sugerencias contenidas en el instrumento correspondientes a la ubicación intrahospitalaria del paciente y al tratamiento antimicrobiano inicial. 


\section{Metodología}

Estudio con diseño descriptivo que abarcó una población de 1,809 pacientes hospitalizados con diagnóstico coincidente ingreso-egreso de NAC, en el periodo comprendido entre el 1 de junio de 2009 hasta el 31 de mayo de 2019. El diagnóstico de neumonía, ratificado por los autores, estuvo basado en los criterios clínicos, radiológicos y necrópsicos (de estar presentes) establecidos (Noya \& Moya, 2017; Longo et al., 2014); para su origen comunitario se tuvo en cuenta la condición de ser pacientes no hospitalizados o que no hubieran estado ingresados en un hospital los 14 días previos al inicio de los síntomas (Longo et al., 2014).

\section{Técnicas y procedimientos:}

Los autores revisaron los documentos clínicos de cada paciente, de donde fue obtenida la información relacionada con los siguientes aspectos: si fue estratificado, estrato en que fue clasificado, área del Departamento de urgencias donde fue atendido, sitio donde fue ingresado y tratamiento antimicrobiano inicial recibido. Los autores también realizaron una estratificación a todos los pacientes (estratificación de control), posterior al ingreso, lo que permitió determinar el estrato real de cada enfermo y valorar la calidad de la estratificación inicial. Todos los pacientes fueron clasificados en alguno de los siguientes estratos:

\begin{tabular}{ll}
\hline Estrato IA & $\begin{array}{l}\text { Neumonía ligera sin particularidades sociofamiliares, económicas y de accesibilidad a } \\
\text { los servicios médicos adversas. }\end{array}$ \\
\hline Estrato IB & $\begin{array}{l}\text { Neumonía ligera con particularidades sociofamiliares, económicas y de accesibilidad } \\
\text { a los servicios médicos adversas. }\end{array}$ \\
\hline Estrato IIA & Neumonía moderada con pocas probabilidades de evolución desfavorable. \\
\hline Estrato IIB & Neumonía moderada con altas probabilidades de evolución desfavorable. \\
\hline Estrato IIIA & Neumonía grave con altas probabilidades de recuperación. \\
\hline Estrato IIIB & Neumonía grave con pocas probabilidades de recuperación. \\
\hline
\end{tabular}

Para el cumplimiento del objetivo se analizó el comportamiento de dos indicadores de proceso: el índice de ubicación intrahospitalaria acorde a la sugerencia del IENAC y el índice de tratamiento antimicrobiano inicial acorde a la sugerencia del instrumento, en la totalidad de los pacientes, en cada uno de los estratos (según la estratificación de control) y en cada subgrupo conformado a partir de la realización de la estratificación inicial y su calidad (pacientes no estratificados, mal estratificados, o bien estratificados).

En el procesamiento de la información se utilizó el programa SPSS versión 15.0 para Windows; los resultados se presentan en gráficos, expresados en por cientos. En el anexo 2 se presenta una caracterización numérica de la población estudiada, a partir de la cual fueron calculados los porcentajes presentados.

\section{Definiciones operacionales:}

- Paciente no estratificado: cuando en la orden de ingreso del expediente clínico del paciente no apareció reflejada explícitamente la ubicación del enfermo en alguno de los estratos del IENAC. 
- Paciente mal estratificado: cuando no hubo coincidencia entre el estrato asignado en el Departamento de urgencias y el asignado por los autores en la estratificación de control; en caso contrario, el paciente fue considerado bien estratificado.

- Ubicación intrahospitalaria acorde a la sugerencia del IENAC: se consideró cuando hubo coincidencia entre el sitio inicial de hospitalización (sala convencional o unidad de cuidados intensivos, UCI) y la recomendación del instrumento de estratificación para el estrato asignado al paciente en la estratificación de control.

- Tratamiento antimicrobiano inicial acorde a la sugerencia del IENAC: se consideró cuando hubo coincidencia entre el antimicrobiano inicialmente utilizado y la recomendación terapéutica del instrumento de estratificación para el estrato asignado al paciente en la estratificación de control.

\section{Análisis estadístico.}

Para el análisis estadístico de los resultados se utilizó la razón de productos cruzados (Odds Ratio, OR) y su intervalo de confianza, así como el estadígrafo $\mathrm{Chi}^{2}$ para diferencias de proporciones en algunas situaciones; se aceptó un nivel de significación de 95\% (p <,05).

\section{Consideraciones éticas.}

Dada las características del estudio no fue necesario realizar consideraciones bioéticas particulares. No obstante, el estudio contó con la evaluación y aprobación del comité de ética de la institución. Los resultados que se muestran han sido utilizados en un ámbito estrictamente científico.

\section{Limitaciones del estudio.}

La investigación ha sido realizada teniendo como sujetos de estudio a pacientes atendidos en las condiciones reales de la asistencia médica en un Departamento de urgencias. La mayoría de la información utilizada en la investigación ha sido recogida en esas condiciones, con lo que esto implica en su plena confiabilidad y en su registro apropiado en el documento clínico. Los autores reconocen que la reconstrucción retrospectiva del estado del paciente al ingreso para la estratificación de control, procedimiento clave en el desarrollo de la investigación, implica asumir riesgos inevitables de algún grado de inexactitud que puede haber influido en los resultados.

Adicionalmente, existe la posibilidad de que los médicos hayan utilizado el IENAC y no dejasen constancia del estrato asignado al enfermo en la orden de ingreso del documento clínico, dando lugar a un sesgo de registro.

\section{Resultados}

\section{Comportamiento del índice de ubicación intrahospitalaria acorde a la sugerencia del IENAC}

En sentido general, la ubicación intrahospitalaria acorde a la sugerencia del IENAC mostró un excelente comportamiento $(96 \%)$.

Aunque hubo pacientes pertenecientes a los estratos IIB (neumonía moderada y altas probabilidades de evolución desfavorable) y IIIB (neumonía grave y pocas probabilidades de recuperación) que fueron incorrectamente ingresados en UCI, el valor más bajo del indicador fue observado en los pacientes del estrato IIIA (neumonía grave y altas probabilidades de recuperación): el 18\% de estos 
casos no fue ubicado acorde a la sugerencia del IENAC, lo cual significa que no ingresó en salas de cuidados intensivos (figura 1). La frecuencia de ubicación no acorde a la sugerencia del instrumento de estratificación en este estrato fue significativamente superior cuando se comparó con el conjunto del resto de los estratos (OR: 6,8[3,5;13,2]).

Figura 1.

Índice de ubicación acorde a la sugerencia del IENAC (en \%) según estratos.

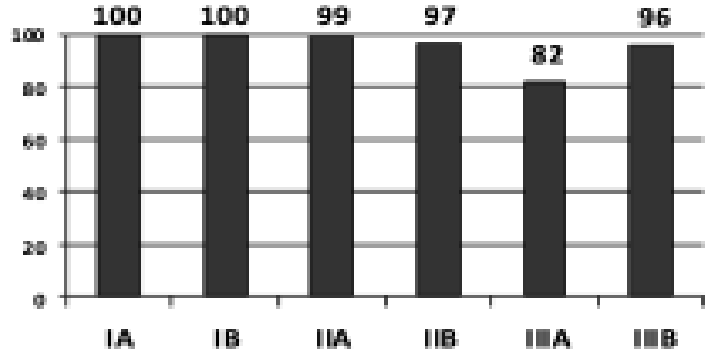

Se constató una menor frecuencia de ubicación intrahospitalaria acorde a la sugerencia del IENAC tanto en los pacientes no estratificados inicialmente $(92 \%)$ como en los mal estratificados (91\%), cuando se comparó con los pacientes bien estratificados $(98 \%)$, lo cual resultó significativo en ambas comparaciones (no estratificados: OR: 5,7 [2,9;10,8]; mal estratificados: OR: 6,1 [3,3;11,1]).

En este mismo análisis, pero particularizado por estratos (obviando lo relativo a los estratos de la neumonía ligera, dado el escaso número de pacientes), resaltan los siguientes resultados (figura 2):

Figura 2.

Índice de ubicación acorde a la sugerencia del IENAC (en \%) según estratos y subgrupos conformados a partir de la realización y calidad de la estratificación.

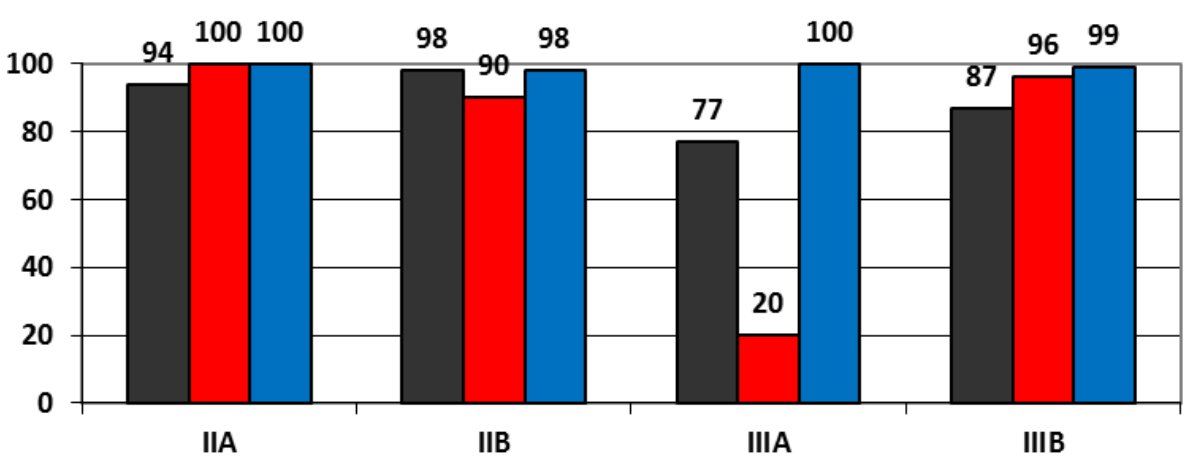

ano estratificados $\square$ Mal estratificados $\square$ Bien estratificados

- En el estrato IIA, el único paciente con ubicación no acorde a la sugerencia del IENAC no fue inicialmente estratificado.

- En el estrato IIB el valor más bajo del índice fue constatado en los pacientes mal estratificados, lo cual fue significativo estadísticamente cuando se comparó con los bien estratificados (OR: $6,5[3,4 ; 12,5])$.

- Todos los pacientes del estrato IIIA que no fueron ubicados en UCI pertenecían a los subgrupos de no estratificados o mal estratificados; en este último subgrupo, la ubicación no acorde a la 
sugerencia del instrumento de estratificación representó el 80\% de los pacientes, mientras que todos los pacientes bien estratificados ingresaron en UCI. Se observó una asociación significativa entre la no estratificación inicial y la ubicación no acorde a la sugerencia del IENAC $\left(\mathrm{X}^{2}=5,8\right.$ $\mathrm{p}<, 05)$, y entre la estratificación inicial incorrecta con la ubicación no acorde a la sugerencia del IENAC $\left(\mathrm{X}^{2}=19,1 \mathrm{p}<, 05\right)$.

- En el estrato IIIB se mantuvo el predominio de pacientes ubicados acorde a la sugerencia del IENAG en el subgrupo de pacientes bien estratificados, comparado con los subgrupos de no estratificados y mal estratificados. En este estrato se comprobó una asociación estadísticamente significativa entre la no estratificación inicial y la ubicación no acorde a la sugerencia del IENAC (OR: $12,9[2,6 ; 64,4])$.

\section{Comportamiento del índice de tratamiento antimicrobiano inicial acorde a la sugerencia del IENAC}

Diferente situación fue observada en cuanto a la correspondencia del tratamiento antimicrobiano inicial con la propuesta del instrumento de estratificación, la cual solo fue del $61 \%$.

Los dos estratos correspondientes a la neumonía ligera mostraron los valores más bajos de tratamiento antimicrobiano inicial acorde a la sugerencia del IENAC (figura 3).

Figura 3.

Índice de tratamiento antimicrobiano inicial acorde a la sugerencia del IENAC (en \%) según estratos.

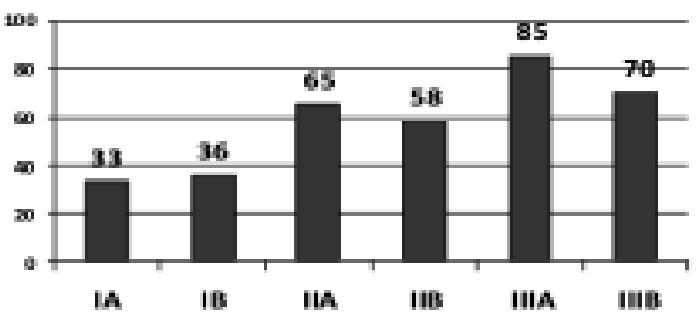

De la misma forma, y aunque con porcentajes más elevados que en la neumonía ligera, este indicador no sobrepasó el 70\% en los estratos de la neumonía moderada (IIA y IIB). Si bien el estrato IIIA mostró el valor más bajo de la ubicación acorde a la sugerencia del IENAC, como ya se señaló, fue el estrato con el valor más elevado de tratamiento antimicrobiano inicial acorde a la sugerencia del IENAC, por encima del 80\%; este resultado también fue estadísticamente significativo cuando se comparó con el conjunto del resto de los estratos (OR: 3,7[1,9;7,2]).

En cuanto al comportamiento de este indicador según los subgrupos conformados a partir de la realización y calidad de la estratificación, se observó un comportamiento más desfavorable del indicador en los pacientes mal estratificados (53\%) comparado con los no estratificados $(69 \%)$ y con los bien estratificados (61\%); hubo significación estadística en las diferencias entre los subgrupos "mal estratificados" y "bien estratificados" (OR: 1,4[1,1;1,8]).

Este análisis, pero teniendo en cuenta los diferentes estratos, no mostró un patrón de comportamiento uniforme (figura 4), aunque es de resaltar que, en los escasos pacientes de los dos estratos de neumonía ligera (estratos IA y IB), casi todos los inicialmente mal estratificados fueron tratados no acorde a la sugerencia del IENAC. Adicionalmente, fueron observadas diferencias estadísticamente significativas 
en la comparación entre los subgrupos "mal estratificados" y "bien estratificados" correspondientes al estrato IIB (OR: $1,3[1 ; 1,8])$.

\section{Figura 4.}

Índice de tratamiento antimicrobiano inicial acorde a la sugerencia del IENAC (en \%) según estratos y subgrupos conformados a partir de la realización y calidad de la estratificación.

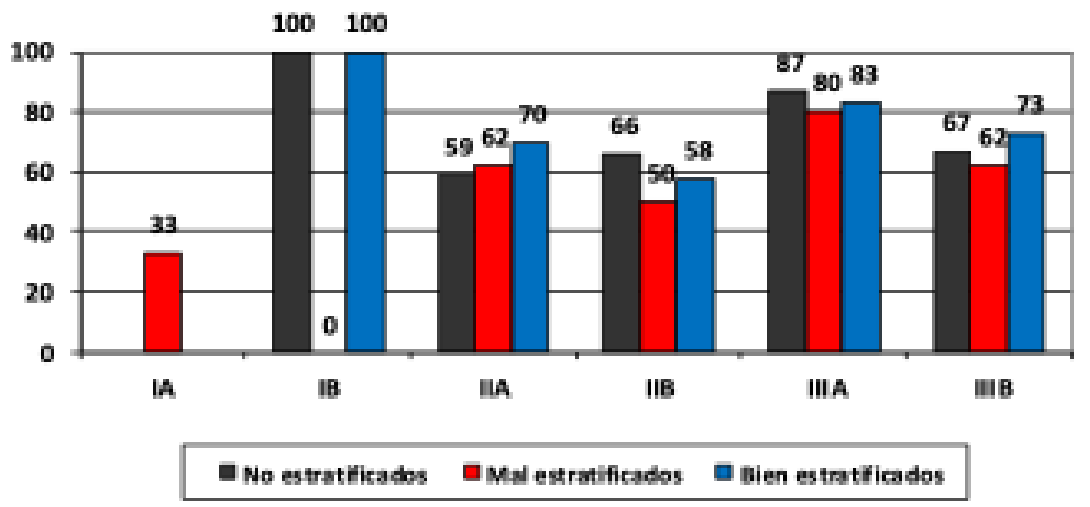

\section{Discusión}

Para analizar los resultados relacionados con el comportamiento del índice de ubicación intrahospitalaria acorde a las sugerencias del instrumento de estratificación debemos recordar, primeramente, que solo los pacientes clasificados como neumonía grave y altas probabilidades de recuperación (estrato IIIA) deben ser ingresados en una UCI, según el IENAC. Para el resto de los pacientes, lo que incluye a los enfermos graves, pero con pocas probabilidades de recuperación, la sugerencia de ubicación es la sala de cuidados convencionales.

Lo anterior es un primer elemento que exlica por qué, a priori, se esperaba un buen comportamiento general del indicador relacionado con la ubicación de los pacientes.

El excelente cumplimiento por los médicos asistenciales de lo sugerido en el IENAC con respecto a la ubicación del paciente estuvo garantizado por el franco predominio de pacientes enmarcados en los estratos IIB y IIIB, cuya suma abarcó al 88\% de la población de la serie; en cambio, los pacientes del estrato IIIA, tributarios del ingreso en la UGI, solo representaron el $4 \%$ del total de pacientes. Y es precisamente la cuestión relacionada con la admisión o no del paciente con NAC en una UCI el dilema más serio a que se enfrentan los médicos en un Departamento de urgencias (Lim et al., 2003; Huijts et al., 2014); razón por la cual han sido diseñadas la mayoría de las escalas pronósticas utilizadas en la actualidad (Fine et al., 1997; Torres et al., 2013; Leoni \& Rello, 2017; Weir et al., 2015; Yeon et al., 2016; Delgado, 2013).

Aunque algunos pacientes pertenecientes a los estratos IIB y IIIB fueron equivocadamente ubicados en la UCI (lo cual los autores consideran un "error por exceso"), el resultado más importante radica en la ubicación en sala convencional de hospitalización (o sea, fuera de la UCI), del 18\% de los pacientes clasificados en el estrato IIIA según la estratificación de control. Esta ubicación implica la privación, para estos enfermos, de la posibilidad de recibir una asistencia médica y cuidados de enfermería particulares en correspondencia con el estado de gravedad con que cursa su proceso neumónico.

El análisis del comportamiento del indicador correspondiente a la ubicación del paciente, teniendo en cuenta en dicho análisis la realización y calidad del proceso de estratificación, puso de manifiesto la 
estrecha relación entre la no estratificación del paciente con NAG en el Departamento de urgencias, o la incorrecta estratificación, con la ubicación intrahospitalaria no acorde con las sugerencias del instrumento de estratificación utilizado.

Si particularizamos en el estrato que presentó el comportamiento más bajo del indicador relacionado con la ubicación del paciente (el estrato IIIA), queda evidenciado que todos los pacientes correctamente clasificados en este estrato fueron ubicados en la UCI. En cambio, resultó llamativa la diferencia observada en el cumplimiento del indicador entre el subgrupo de pacientes no estratificados en el Departamento de urgencias y el subgrupo de pacientes que fueron incorrectamente estratificados: en los primeros el incumplimiento fue observado solo en el $23 \%$, mientras que en los segundos dicho incumplimiento abarcó al 80\% de los casos.

Este resultado parece corroborar un hecho observado: los profesionales actuantes en el área roja del Departamento de urgencia, en su totalidad médicos intensivistas, tienden a no registrar el estrato del paciente en el documento clínico (criterio operativo utilizado en la investigación para considerar al paciente como estratificado), pero siguen las conductas establecidas en sus normas y guías con los pacientes en estado de gravedad, que es lo más importante en el orden práctico. Siguiendo esta línea de pensamiento, también es posible que algunos de los pacientes del estrato IIIB (graves con pocas probabilidades de recuperación) que no fueron inicialmente estratificados y que fueron ubicados en la UCI, hayan sido atendidos directamente, por su estado de gravedad, en el área roja del Departamento de urgencias.

En cuanto al cumplimiento de las sugerencias del IENAG relacionadas con el tratamiento antimicrobiano inicial resultó llamativo el bajo cumplimiento de lo propuesto en el instrumento en los estratos correspondientes a la neumonía ligera y a la neumonía moderada; así como el aceptable cumplimiento de la antibioticoterapia inicial en los pacientes con neumonía grave, fundamentalmente en aquellos con elevadas probabilidades de recuperación (estrato IIIA), y que resultó contrastante con lo observado para este estrato con respecto a la ubicación intrahospitalaria.

En plena consonancia con la política establecida en la institución para el uso de los antimicrobianos, y en correspondencia con el cuadro básico de medicamentos del país, el instrumento de estratificación utilizado en los pacientes con NAC deja explícitamente declarada cual es la propuesta del antibiótico empírico a utilizar inicialmente en dependencia de la magnitud de la afección, siempre que no existan particularidades que requieran antibióticos específicos (Saldías et al., 2018; Monedero et al., 2016; Montero et al., 2017; Pakhale et al., 2014; Postma et al., 2015; González et al., 2014; Geijo et al., 2014; Obed et al., 2014; GBD 2015 LRI Collaborators, 2017; Torres et al., 2014).

Esta propuesta terapéutica transita por la utilización oral de amoxicilina con azitromicina en la afección ligera (estratos IA y IB); cefalosporina de segunda generación (cefuroxima) o amoxicilina protegida (amoxicilina más sulbactám) por vía parenteral, también combinado con macrólido, en la neumonía moderada (estratos IIA y IIB); y reserva el empleo de cefalosporinas de tercera generación (ceftriaxona y cefotaxima) para los pacientes con una afectación severa (estratos IIIA y IIIB), siempre combinado con azitromicina. En estos últimos estratos se deja abierta la posibilidad de utilización de cefuroxima o amoxicilina más sulbactám, pero duplicando las dosis propuestas para los pacientes con neumonía moderada.

Aunque la falta de sistematicidad en la disponibilidad de los medicamentos propuestos por el IENAC (derivada fundamentalmente de la injusta guerra económica que se nos impone) ha conducido en determinados momentos al empleo de otros antibióticos alternativos, la principal causa del insuficiente comportamiento del índice de tratamiento antimicrobiano inicial acorde a la sugerencia del IENAC 
radica en la extendida utilización de las cefalosporinas de tercera generación (específicamente la ceftriaxona) en muchos procesos infecciosos bacterianos, y en la neumonía entre estos.

En el caso concreto de nuestra serie, a través de la revisión de las indicaciones terapéuticas individuales de los pacientes, se comprobó una amplia utilización de ceftriaxona como antimicrobiano inicial en la mayoría de los pocos pacientes con neumonía ligera hospitalizados, y en gran parte de los pacientes con neumonía moderada; situación esta que incluye al estrato más numeroso de toda la población estudiada: el estrato IIB. Esquemas antimicrobianos puntuales a algunos pacientes, con empleo a la admisión de antibióticos como vancomicina, meropenem o cefepime, explican el porcentaje de pacientes del estrato IIIA que no fueron tratados acorde a la sugerencia del instrumento de estratificación.

De cualquier manera, y sin profundizar excesivamente en la repercusión de la situación antes mencionada, las consecuencias evidentes del insuficiente comportamiento del indicador "índice de tratamiento antimicrobiano inicial acorde a la sugerencia del IENAC" están enmarcadas en el área económica (costos hospitalarios), en el área administrativa (vulneración de la política antimicrobiana de la institución) y en una posible contribución al nefasto fenómeno universal de la resistencia a los antibióticos.

En el análisis del comportamiento del índice de tratamiento antimicrobiano inicial acorde a la sugerencia del IENAC teniendo en cuenta los subgrupos conformados a partir de la realización y calidad de la estratificación, se comprobó una evidente relación entre la calidad de la estratificación y el tratamiento no acorde al instrumento; tanto para la población total estudiada como para el estrato más numeroso de la serie, el de los pacientes con neumonía moderada y altas probabilidades de evolución desfavorable (estrato IIB).

Como conclusiones del estudio los autores reconocen que un elevado desempeño en el cumplimiento de la sugerencia de ubicación intrahospitalaria ha sido una de las características del proceso de utilización del IENAC en la estratificación del paciente con NAC en los últimos diez años; como elemento negativo se destaca el frecuente incumplimiento de la sugerencia de tratamiento antimicrobiano inicial.

En segundo lugar, se concluye que los resultados obtenidos permiten asumir, en la serie estudiada, la existencia de una relación de evidente importancia práctica entre la no estratificación o estratificación incorrecta del paciente por una parte, y la ubicación intrahospitalaria del enfermo no acorde a la sugerencia del instrumento empleado, por la otra; asociación que también se presentó entre la calidad de la estratificación y el cumplimiento de la sugerencia de tratamiento antimicrobiano inicial.

\section{Aspectos éticos}

El estudio contó con la evaluación y aprobación del comité de ética de la institución.

\section{Conflicto de interés}

Los autores declaran no tener ningún conflicto de interés relacionado directa o indirectamente con los contenidos del manuscrito. 


\section{Financiamiento}

La presente investigación no ha recibido ayudas específicas provenientes de agencias del sector público, sector comercial o entidades sin ánimo de lucro.

\section{Agradecimientos}

Se agradece a los doctores Sandy Mederos Sust, Pedro Luis Ocampo Miralles, Jorge Labrada González, Adan Villamizar Sánchez, Erardo Labrada Ortiz, Edwin Ojeda Rodríguez y Duniel Sánchez Medina su contribución a la recogida de la información en diferentes momentos de la investigación.

\section{Referencias}

Aleaga-Hernandez, Y.Y., Serra-Valdes, M.A., \& Cordero-López, G. (2015). Neumonía adquirida en la comunidad: aspectos clínicos y valoración del riesgo en ancianos hospitalizados. Revista Cubana de Salud Pública, 41(3):413-26.

Báez-Saldaña, R., Gómez-Zamora, G., López-Elizondo, C., Molina-Corona, H., Santillán-Martínez, A., \& Sánchez-Hernández,J. (2013). Neumonía adquirida en la comunidad. Revisión y actualización con una perspectiva orientada a la calidad de la atención médica. Neumol Cir Torax.,72(1):6-43.

Calle, A., Márquez, M.A., Arellano, M., Pérez, L., Figueras, M.P., \& Miralles, R. (2014). Valoración geriátrica y factores pronósticos de mortalidad en pacientes muy ancianos con neumonía extrahospitalaria. Arch Bronconeumol., 50(10):429-34. https://doi.org/10.1016/j. arbres.2014.01.012

Corona-Martínez, L.A., González-Morales, I., Fragoso-Marchante, M.C., Mederos-Sust, S., Hernández-Abreu, C., \& Borroto-Lecuna, S. (2015). Experiencias en la aplicación de un instrumento para la estratificación de pacientes con neumonía adquirida en la comunidad. Rev Cubana Med. [Internet];54(4). Disponible en: http://bvs.sld.cu/revistas/med/vol54_4_15/med03415.htm

Delgado, M. (2013). Uso rutinario del Pneumonia Severity Index en el servicio de urgencias: efecto sobre los indicadores de proceso y resultado en neumonia adquirida en la comunidad. Enferm Infecc Microbiol Clin.,31(5):289-97. https://doi.org/10.1016/j.eimc.2012.04.012

Falguera, M.F., \& Ramírez, R. (2015). Neumonía adquirida en la comunidad. Rev Clin Esp.,215(8):45867. https://doi.org/10.1016/j.rce.2015.06.002

Fine, M.J., Auble, T.E., Yealy, D.M., Hanusa, B.H., Weissfeld, L.A., \& Singer D.E. (1997). A prediction rule to identify low-risk patients with community-acquired pneumonia. N Engl J Med., 336:243250. https://doi.org/10.1056/nejm199701233360402

GBD 2015 LRI Collaborators. (2017). Estimates of the global, regional, and national morbidity, mortality, and aetiologies of lower respiratory tract infections in 195 countries: a systematic analysis for the Global Burden of Disease Study 2015. Lancet Infect Dis., 17:1 133-61. https://doi. org/10.1016/S1473-3099(18)30310-4

Geijo, M., Bermejo, E., \& García, A. (2014). Protocolo diagnóstico y terapéutico de las neumonías extrahospitalarias. Medicine., 11(52):3076-80. https://doi.org/10.1016/S0304-5412(14)70742-5 
González del Castillo, J., Linares, M.J.P., Menéndez, R., Mujal, A., Navas, E., \& Barberán, A. (2014). Guía de consenso para el abordaje de la NAC en al paciente anciano. Rev Esp Geriatr Gerontol., 49(6:)279-91. https://doi.org/10.1016/j.regg.2014.04.002

Huijts, S.M., Boersma, W.G., Grobbee, D.E., Gruber, W.C., Jansen, K.U., \& Kluytmans, J.W. (2014). Predicting pneumococcal community-acquired pneumonia in the emergency department: evaluation of clinical parameters. Clin Microbiol Infect., 20:1316-22. https://doi.org/10.1111/14690691.12740

Huerta, A., Crisafulli, E., Menéndez, R., Martinez, R., Soler, N., \& Guerrero, M. (2013). Pneumonia and nonpneumonia exacerbations of COPD: inflamatory response and clinical characteristics. Chest., 144:1134-42. https://doi.org/10.1378/chest.13-0488

Julián-Jiménez, A., Adán-Valero, I., Beteta-López, A., Cano-Martín, L.M., Fernández-Rodríguez, O., \& Rubio-Díaz, R. (2018). Recomendaciones para la atención del paciente con neumonía adquirida en la comunidad en los Servicios de Urgencias. Rev Esp Quimioter., 31(2):186-202. https://www. ncbi.nlm.nih.gov/pmc/articles/PMC6159381/

Leoni, D., \& Rello, J. (2017). Severe community-acquired pneumonia: optimal management. Curr Opin Infect Dis., 30(2):240-7. https://doi.org/10.1097/qco.0000000000000349

Lim, W.S., Van der Eerden, M.M., Laing, R., Boersma, W.G., Karalus, N., \& Town, G.I. (2003). Defining community acquired pneumonia severity on presentation to hospital: an international derivation and validation study. Thorax., 58:377-382. https://doi.org/10.1136/thorax.58.5.377

Longo, D.L., Musher, D.M., Thoner, A.R., \& Debakey, M.E. (2014). Community acquired pneumonia. N EnglJ Med., 371:1619-28. https://doi.org/10.1056/nejmra1312885

Martínez-Vernaza, S., Mckinley, E., Soto, M.J., \& Gualtero S. (2018). Neumonía adquirida en la comunidad: una revisión narrativa. Univ Med. 2018 [Internet]. 59(4). Disponible en: https://doi. org/10.11144/Javeriana.umed59-4.neum

Ministerio de Salud Pública. (2019). Anuario estadístico de Salud. La Habana: Dirección Nacional de Registros Médicos y estadísticas del Minsap; Disponible en: http://bvscuba.sld.cu/anuarioestadistico-de-cuba/

Monedero Mira MJ, Sales MB, Domingo CG, et al. Tratamiento empírico de las infecciones del adulto. FMC. 2016;23:9-71. https://doi.org/10.1016/j.fmc.2015.12.002

Montero-Solano, G., Hernández-Romero, G., Vega-Chaves, J.C., \& Ramírez-Cardoce, M. (2017). Manejo de la neumonía adquirida en la comunidad en el adulto mayor. Rev Cl EMed UCR., 7(2):11-20. https://doi.org/10.15517/rc_ucr-hsjd.v7i2.29141

Noya-Chaveco, M.E., \& Moya-González, N.L. (2017). Parte VII. Enfermedades del sistema respiratorio. Capítulo 31. Neumopatías inflamatorias agudas no tuberculosas. Roca Goderich. Temas de Medicina Interna. Tomo I. Quinta edición. 5 ed. La Habana, GU: EGIMED. Disponible en: http://bvs.sld.cu/libros_texto/ roca_temas_medicina_interna_tomol_quintaedicion/ cap31. pdf

Obed, M., García-Vidal, C., Pessacq, P., Mykietiuk, A., Viasus, D., \& Cazzola, L. (2014). Características clínicas y pronóstico de la neumonía adquirida en la comunidad causada por Staphylococcus aureus resistente a la meticilina. Enferm Infecc Microbiol Clin., Jan;32(1):23-7. https://doi. org/10.1016/j.eimc.2013.01.006 
Pakhale S, Mulpuru S, Verheij TJM, Kochen MM, Rohde GGU, Bjerre LM. Antibiotics for community-acquired pneumonia in adult outpatients. Cochrane Database of Systematic Reviews 2014, Issue 10. Art. No.: CD002109. https://doi.org/10.1002/14651858.CD002 109.pub4

Postma,D.F., Van Werkhoven, C.H., Van Elden, L., Thijsen, S., Hoepelman, A., \& Kluytmans, J. (2015). Antibiotic treatment strategies for community acquired pneumonia in adults. N Engl J Med., 372:1312-23. https://doi.org/10.1056 / NEJMoa1406330

Saldías-Peñafiel, F., Gassmann-Poniachik, J., Canelo-López, A., \& Díaz-Patiño, O. (2018). Características clínicas de la neumonía adquirida en la comunidad del adulto inmunocompetente hospitalizado según el agente causal. Rev Med Chile., 146:1371-83. http://dx.doi.org/10.4067/ s0034-98872018001201371

Spoorenberg, S.M.G., Bos, W.J.W., \& Heijligenberg, R. (2014). Microbial aetiology, outcomes, and costs of hospitalisation for community-acquired pneumonia; an observational analysis. BMC Infect Dis., 14:335-8. https://doi.org/10.1186/1471-2334-14-335

Torres, A., Barberán, J., Falguera, M., Menéndez, R., Molina, J., \& Olaechea, P. (2013). Guía multidisciplinar para la valoración pronóstica, diagnóstico y tratamiento de la neumonía adquirida en la comunidad. Med Glin (Barc)., 140:223,e 1-19. https:/ /doi.org/10.1016/j.medcli.2012.09.034

Torres, A., Blasi, F., Peetermans, W.E., Viegi, G., \& Welte, T. (2014). The aetiology and antibiotic management of community-acquired pneumonia in adults in Europe: a literature review. Eur J Clin Microbiol Infect Dis., 33:1065-79.

Weir, D.L., Majumdar, S.R., McAlister, F.A., Marrie, T.J., \& Eurich, D.T. (2015).The impact of multimorbidity on short-term events in patients with community-acquired pneumonia: Prospective cohort study. Clin Microbiol Infect., 21:264.e7-264.e13. https://doi.org/10.1016/j. cmi.2014.11.002

Yeon-Lee, S., Cha, S.I., Seo, H., Oh, S., Choi, K.J., \& Yoo, S.S. (2016). Multimarker Prognostication for Hospitalized Patients with Community-acquired Pneumonia. Intern Med., 55(8):887-93. https://doi.org/10.2169/internalmedicine.55.5764

\begin{abstract}
The initial evaluation of the patient's condition with pneumonia is a very important assistance action. The objective was evaluate the relationship between the frequency and quality of the stratification process of the patient with pneumonia, and the execution of suggestions of intrahospitalary location and the initial antimicrobial treatment of stratification instrument. A descriptive study was done on a population of 1,809 patients hospitalized during 10 years. The indexes of intrahospitalary location and of antimicrobian initial treatment were analized according to the suggestions of the instrument; in the statistical analysis it was used the odds ratio and the statistician $\mathrm{X}^{2}$, with a significant level of $95 \%$. The intrahospitalary location was in agreement with the suggestion of the instrument in $96 \%$ of the cases, with the lowest value in patients with serious pneumonia and high recovery probabilities $(82 \%, \mathrm{p}<, 05)$. The frequency of intrahospitalary location was bigger and veryfied with the suggestion of the instrument in the termed well stratified patients $(\mathrm{p}<, 05)$, fundamentally in the patients with serious pneumonia and high recovery probabilities. The correspondence of the initial antimicrobial treatment with the proposal of the instrument was of $61 \%$; the stratum IIIA showed the highest value $(80 \%, \mathrm{p}<, 05)$.
\end{abstract}


As conclusions, a high performance in the execution of the suggestion of the intrahospitalary location has been one of the characteristics of the process, although as a negative element it stands out the frequent non-fulfillment of the suggestion of the initial antimicrobial treatment. There was a relationship between the stratification process and the execution of the suggestion of the intrahospitalary location.

Keywords: community-acquired infection; Hospital admittance; Pneumonia 
Anexo 1

Instrumento de estratificación del paciente con neumonía adquirida en la comunidad (IENAC)

CLASE I (neumonía ligera)
CLASE II (neumonía moderada)
CLASE III (neumonía grave o severa)

Categoría A (sin particu- Categoría A (con pocas Categoría A (con altas problares sociofamiliares adver- probabilidades de evolu- abilidades de recuperación) sas)

Contexto del paciente:

- Menor de 60 años.

- Buen estado general.

- No síntomas de compromiso funcional respiratorio.

- No derrame pleural.

- Discreto infiltrado pulmonar circunscrito a un lóbulo.

- No enfermedades crónicas asociadas o respiratorias previas.

- Condiciones sociales, económicas y accesibilidad a los servicios médicos favor-

ables.
Categoría B (con particu- Categoría B (con altas prob- Categoría B (con escasas lares sociofamiliares adver- abilidades de evolución des- probabilidades de recusas) favorable)

Contexto del paciente:

- Mayor de 60 años.

- Buen estado general.

- No síntomas de compromiso funcional respiratorio.

- No derrame pleural.

- Discreto infiltrado pulmonar circunscrito a un lóbulo.

- Condiciones sociales, económicas y accesibilidad a los servicios médicos desfavorables. peración)

Contexto del paciente:

- Igual a la categoría A, salvo en lo siguiente:

- Enfermedad de base asociada: demencia con validismo funcional deteriorado; insuficiencia cardiaca clase funcional IV; IRC grado $\mathrm{V}$ no en diálisis; insuficiencia hepática crónica avanzada, cáncer avanzado, retraso mental severo; encamamiento irreversible.

\section{ción desfavorable)}

Contexto del paciente:

- Menor de 60 años.

- Moderada toma del estado general.

- No síntomas de compromiso funcional respiratorio, no existe afectación de la conciencia y no hay descompensación cardiovascular.

- Si hay derrame pleural, es de pequeña cuantía.

- En la radiografía de tórax no • hay afectación de más de un lóbulo.
Contexto del paciente:

- Cualquier edad.

- Marcada toma del estado general.

- Síntomas y signos de compromiso funcional respiratorio; existe afectación de la conciencia.

- En la radiografía de tórax afectación de más de un lóbulo, derrame pleural de mediana o gran cuantía.

- Enfermedad de base: ninguna de las consideradas en la categoría B.
Contexto del paciente:

- Cualquier edad.

- No síntomas de compromiso funcional respiratorio y no existe afectación de la conciencia.

- Derrame pleural de mediana cuantía, pero sin compromiso funcional.

- En la radiografía de tórax puede haber afectación de más de un lóbulo.

- Enfermedades crónicas renales, cardiacas, hepáticas o respiratorias previas, ligeramente descompensadas o con altas probabilidades de descompensación. 
ESTRATO IA

Área de atención: amarrilla

Conducta: manejo ambulatorio

ESTRATO IB

Área de atención: amarrilla

Conducta: ingreso en sala

convencional

\section{ESTRATO IIA}

Área de atención: amarrilla

Conducta: ingreso en sala

convencional

\section{ESTRATO IIB}

Área de atención: amarrilla

Conducta: ingreso en sala

convencional
ESTRATO IIIA

Área de atención: roja

Conducta: ingreso en UCI

ESTRATO IIIB

Área de atención: roja

Conducta: ingreso en sala convencional
CLASE I (neumonía ligera)
- Amoxicillina 500 mg-1 g cada - Cefuroxima 750 mg-1.5 8 horas v/o o Cefalexina 500

mg cada 6 horas v/o

más

- Azitromicina 250-500 mg cada 12 horas v/o.

Alternativo

- Penicilina Cristalina 1 millón

Uds cada 6 horas.
GLASE II (neumonía moderada)

\section{g cada 8 horas IM o EV}

o Amoxicilina-sulbactám
GLASE III (neumonía grave o severa)

- Ceftriaxona 1 g cada 12 horas EV solo asociado a Azitromicina $250 \mathrm{mg}$ cada 12 (Trifamox) $750 \mathrm{mg}$ cada 8 horas horas v/o.

EV más

- Azitromicina $250 \mathrm{mg}$ cada 12 horas v/o.
- Cefotaxima $1 \mathrm{~g}$ cada 8 horas

EV asociado a Azitromicina
$250 \mathrm{mg}$ cada 12 horas v/o.

- Amoxicillina-sulbactám

(Trifamox) $1.5 \mathrm{~g}$ cada 8 horas

EV asociado a Azitromicina 250

mg cada 12 horas v/o.

\section{Anexo 2}

Garacterización numérica de la población estudiada según términos e indicadores relacionados con la realización de la estratificación, calidad del proceso e indicadores de cumplimiento de sugerencias del IENAG en cuanto a ubicación y tratamiento antimicrobiano

Frecuencia de los pacientes con ubicación intrahospitalaria acorde a la sugerencia del IENAC.

\begin{tabular}{ccccc}
\hline Estrato & No estratificados & Mal estratificados & Bien estratificados & Total \\
\hline IA & - & 3 de 3 & - & 3 de 3 \\
IB & 2 de 2 & 7 de 7 & 2 de 2 & 11 de 11 \\
IIA & 16 de 17 & 58 de 58 & 53 de 53 & 127 de 128 \\
IIB & 132 de 135 & 193 de 215 & 973 de 990 & 1,298 de 1,340 \\
IIIA & 30 de 39 & 1 de 5 & 29 de 29 & 60 de 73 \\
IIIB & 47 de 54 & 23 de 24 & 174 de 176 & 244 de 254 \\
Todos & 227 de 247 & 285 de 312 & 1,231 de 1,250 & 1,743 de 1,809 \\
\hline
\end{tabular}

Frecuencia de los pacientes con tratamiento antimicrobiano inicial acorde a la sugerencia del IENAC.

\begin{tabular}{ccccc}
\hline Estrato & No estratificados & Mal estratificados & Bien estratificados & Total \\
\hline IA & - & 1 de 3 & - & 1 de 3 \\
IB & 2 de 2 & 0 de 7 & 2 de 2 & 4 de 11 \\
IIA & 10 de 17 & 36 de 58 & 37 de 53 & 83 de 128 \\
IIB & 89 de 135 & 108 de 215 & 574 de 990 & 771 de 1,340 \\
IIIA & 34 de 39 & 4 de 5 & 24 de 29 & 62 de 73 \\
IIIB & 36 de 54 & 15 de 24 & 128 de 176 & 179 de 254 \\
Todos & 171 de 247 & 164 de 312 & 765 de 1,250 & 1,100 de 1,809 \\
\hline
\end{tabular}

